\title{
L'éducation complexe : entretien avec Edgar Morin
}

The complex education : interview with Edgar Morin

\section{Guy Berger, Augustin Mutuale, Fabienne Serina-Karsky et Séverine Parayre}

\section{(2) OpenEdition}

Journals

Édition électronique

URL : http://journals.openedition.org/trema/6193

DOI : $10.4000 /$ trema.6193

ISSN : 2107-0997

Éditeur

Faculté d'Éducation de l'université de Montpellier

Référence électronique

Guy Berger, Augustin Mutuale, Fabienne Serina-Karsky et Séverine Parayre, «L'éducation complexe : entretien avec Edgar Morin », Tréma [En ligne], 54 | 2020, mis en ligne le 18 novembre 2020, consulté le 14 décembre 2020. URL : http://journals.openedition.org/trema/6193 ; DOI : https://doi.org/10.4000/ trema.6193

Ce document a été généré automatiquement le 14 décembre 2020.

Trema 


\section{L'éducation complexe : entretien avec Edgar Morin}

The complex education : interview with Edgar Morin

Guy Berger, Augustin Mutuale, Fabienne Serina-Karsky et Séverine Parayre

\section{Introduction}

1 Ce mardi 26 Novembre 2019, nous avons rendez-vous à 14 heures avec Edgar Morin dans ses bureaux de l'Institut Botanique de Montpellier, ville dans laquelle il s'est installé durant l'été caniculaire de 2018. Notre petit groupe composé de quatre chercheurs parisiens s'attelle aux derniers préparatifs dans le TGV : nous relisons, répétons, discutons, imaginons, bref, nous continuons à chercher ensemble ce qui nous parait constituer l'apport majeur de la pensée complexe à l'éducation. Préparé pendant plusieurs séances de travail et ayant donné lieu à de nombreuses lectures et discussions, cet entretien est pour nous l'occasion d'instaurer un dialogue avec le Maitre de la complexité. Guy Berger sera notre porte-parole. Nous voici arrivés dans cet endroit au charme surrané, en face du Jardin des Plantes. La porte s'ouvre, l'assistante d'Edgar Morin nous accueille et nous laisse nous installer' ${ }^{1}$. La porte s'ouvre...

\section{Entretien}

FABIENNE SERINA-KARSKY : Cher Edgar, je voulais tout d'abord vous remercier infiniment de nous accorder un peu de votre temps et de nous recevoir tous les quatre dans ce lieu, à I'Institut Botanique de Montpellier où se trouvent vos bureaux. Nous avons communiqué plusieurs fois pour préparer cet entretien pour la revue Tréma et le numéro spécial que nous consacrons à ce que nous nommons l'Education complexe par lequel nous souhaitons souligner votre apport, avec la pensée complexe, à l'éducation. Guy Berger nous a fait l'amitié de bien vouloir travailler avec nous pour préparer cet entretien, c'est lui qui partagera cette conversation avec vous. Après vous avoir resituer son parcours et les rencontres qui l'ont mené jusqu'à vous, Guy Berger introduira des questions liées à la notion 
de complexité à l'épreuve des conceptions éducatives telles que vous les avez travaillées à de nombreuses reprises.

GUY BERgER : Tout d'abord merci d'avoir accepté de nous recevoir. Je viens aussi non seulement par rapport à une amitié qui n'est pas seulement à votre égard, mais à l'égard d'Ardoino dont je fus le compagnon de travail pendant très longtemps, de Christiane Peyron Bonjan² que nous avons évoqué il y a un instant. J'avais été aussi l'éditeur d'un livre sur un «Vocabulaire de la complexité », votre vocabulaire, réalisé par Marius Mukungu Kakangu dont nous nous sommes entretenus ${ }^{3}$. J'ai avec vous beaucoup de liens. Nous nous sommes vus à des tables rondes, je me souviens à La Villette où nous participions ensemble, nous avons été ensemble membres de jurys, mais je voudrais dire beaucoup mieux pourquoi personnellement vous avez beaucoup compté pour moi. Je le ferai à partir d'un récit.

Jeune assistant de psycho, après avoir eu une formation de philosophe, j'avais été sollicité par la télévision scolaire, pour accompagner une expérience massive, de création d'un collège expérimental qui serait centré sur l'audiovisuel, avec comme idée centrale de la part des professeurs, de vérifier si une utilisation systématique de l'image et d'un système de culture de masse avait une action possible sur les modes d'apprentissage des enfants de sixième et des classes suivantes, mais surtout si cela pouvait agir sur les difficultés socioculturelles qu'on pensait liées au langage et à l'écrit, et dont on imaginait qu'elles seraient moins profondes à l'égard de l'image. J'étais chargé d'élaborer puis de conduire un programme de recherches, ça a été un peu mon Plozévet, avec un assistant de sémiologie, un sociologue, des psycho-sociologues, des psychomotriciens etc, pour suivre cette expérience qui a duré une dizaine d'années. Et très rapidement on s'est rendu compte que c'est autre chose qui se passait. On était parti d'un modèle causaliste, d'un modèle linéaire, selon lequel l'image entraînerait d'autres types d'apprentissage et on considérait qu'on disposait ainsi d'une variable: l'image et sa durée. Certes on s'était rendu compte qu'il fallait construire un nouveau bâtiment composé d'un jeu de triangles équilatéraux avec des angles de 60 degrés, pour avoir une vision maximum, et que du coup on brisait le modèle de la classe, et on avait des groupes différents, mais quand on a interrogé les élèves au bout de 2 ans, on s'est rendu compte que presqu'aucun ne nous parlait de la télévision. Mais ils nous parlaient beaucoup, d'abord de leurs enseignants, et du changement de ces enseignants. Lorsque ceux-ci rencontraient les réalisateurs, et à l'époque la télévision scolaire avait, à côté des techniciens, des réalisateurs comme Rohmer et comme Dugowson qui sont devenus des gens célèbres, et bien ces réalisateurs leur disaient «bon je sais de quoi tu veux parler, mais qu'est-ce que tu veux obtenir? Est-ce que tu as envie que les enfants discutent, est-ce que tu as envie qu'ils découvrent qu'il y a plusieurs vérités, est-ce que tu as envie qu'ils interprètent des images ? ». Et brusquement les enseignants se rendaient compte qu'ils étaient historiens, littéraires, mathématiciens, mais qu'ils n'avaient pas de MÉtrer d'enseignant. Et qu'en tant qu'enseignants, ils ne savaient pas ce qu'ils voulaient obtenir. Et donc ces enseignants ont dû changer, ils travaillaient ensemble, ils produisaient des images, et ainsi ils acceptaient que les élèves les discutent. Un élève qui dirait à un enseignant, par le seul fait de poser une question, «je n'ai pas compris », est vécu comme agressif par son enseignant, un élève qui dit "cette vidéo est mal fichue, j'y comprends rien, elle est trop rapide », même si cette vidéo est faite par cet enseignant, elle devient un médiateur objectivé. Pendant les visionnements les enseignants d'ailleurs, ne pensent plus à ce qu'ils veulent dire, mais écoutent, ou regardent, comment les élèves réagissent. Et par conséquent les élèves nous ont parlé de l'organisation, de leur rapport entre eux élèves, de la façon dont les enseignants les enseignaient, de la possibilité de les critiquer, de la façon d'être accueilli etc. Avec ceux qui étaient les promoteurs de cette grosse expérience, nous avons découvert, nous avons cru découvrir, qu'en agissant sur un élément, en réalité nous avions changé le système. On l'avait même changé au point qu'un inspecteur pédagogique venu inspecter l'un des enseignants, avait refusé de l'inspecter parce que l'enseignant utilisait une vidéo qui n'était pas son œuvre et il a fallu aller au ministère demander de changer les modalités d'inspection. En changeant les statuts, les modalités, les horaires, les bâtiments etc., on agissait sur le système. Et dans notre innocence, on avait cru avoir fait une découverte, et 
c'est seulement après avoir, dans nos premières conclusions, insisté sur cet aspect systémique de la réalité, qu'on a découvert qu'il existait une théorie des systèmes. C'est à la même époque que paraissent le Macroscope de Rosnay ${ }^{4}$, les premiers travaux de Barel ${ }^{5}$, de Lesourne ${ }^{6}$, etc, bien après Commune en France, la métamorphose de Plodemet ${ }^{7}$ en 1967, Le Paradigme perdu ${ }^{8}$ en 1973.

Le Paradigme perdu, avant même que la notion de complexité prenne chez vous l'importance et la généralité qu'elle a eue, posait déjà un certain nombre de problèmes, celui de ce que vous avez appelé l'importance du modèle biologique, en opposition au modèle physiciste, qui était le nôtre et qui reposait sur la séquence cause/effet, l'importance de ce que vous y appeliez aussi la soudure épistémologique, c'est-à-dire le fait que c'était par la réflexion sur la manière de connaitre qu'on arrivait à comprendre les liens entre les différents moments, ce que vous aviez aussi désigné comme l'hyper-complexité, et par conséquent, à partir de là vous avez été de fait, un de nos éclaireurs, si je puis dire, nous permettant d'analyser les choses, et par certains côtes co-auteur de ce que fut ma carrière puisque après ce travail au collège de Marly le Roi, ou plutôt en même temps, j'ai fait partie de l'équipe fondatrice de I'Université de Vincennes qui allait devenir Paris 8 à Saint-Denis ${ }^{9}$ et qui, dès le départ, était une proposition de système autre, et non une proposition de rénovation ou d'innovation au sens élémentaire de ce terme.

C'est donc avec reconnaissance que je vous rencontre, c'est une manière de vous remercier de ce que vous m'avez appris. Mais il est évident que depuis, votre conception s'est élargie, développée, multipliée et que par certains côtés moi-même, il m'est arrivé d'apprendre quelques petites choses, et c'est là que nous trouvons cette notion et de complexité, mais aussi le fait que vous l'ayez progressivement et de multiples fois mise à l'épreuve des conceptions éducatives. Avant de vous laisser dire ce que vous avez à dire, je voudrais volontiers partir de cette phrase de l'introduction de La tête bien faite ${ }^{10}$ dans lequel vous écrivez ironiquement : " ceux qui ne m'ont pas lu et me jugent selon les ragots du microcosme m'attribuent l'idée bizarre selon laquelle je proposerais une potion magique nommée complexité comme un remède à tous les maux de l'esprit. Au contraire, la complexité est pour moi un défi que j'ai toujours proposé de relever ». Et je pense que ce qui est important pour nous, c'est de montrer comment ces défis de la complexité ont pour vous rejoint les défis de l'éducation.

Edgar Morin : Merci de m'avoir bien situé votre travail et votre itinéraire. Vous vous êtes occupé d'une chose que je n'ai pas traité centralement mais qui est capitale, les relations entre enseignants et enseignés. Cette idée de complexité je la fonde sur deux préliminaires. En premier, je suis persuadé qu'il faut une révolution intellectuelle, c'est à dire dans la manière de connaitre, dans la manière de penser. Pourquoi je pense qu'il faut une révolution intellectuelle: c'est parce que dans la manière de connaitre, la manière de penser que l'on enseigne se fonde effectivement non seulement sur ce que vous avez dit, une causalité linéaire, sur une verticalité, sur une vision découpée et fragmentée du réel et enfermée dans des compartiments de plus en plus épais que sont les disciplines, mais aussi sur des principes, qui sont des principes de réduction d'un tout complexe à ces éléments premiers constituant le tout, qui rendent incapable de concevoir qu'un tout est plus que la somme des parties, incapable de comprendre que la grande originalité du monde et de la vie, c'est que dès que vous avez une organisation à partir d'éléments différents, il se produit des émergences, des qualités nouvelles qui n'existent pas dans les éléments, et des contraintes nouvelles qui n'existent pas.

Donc, si vous voulez, l'autre pensée est de plus en plus infirme devant une réalité que je dis de plus en plus complexe, de plus en plus faite de relations, de rétroactions, de récursions etc. Et si je dis qu'il nous faut une révolution intellectuelle, je dis qu'il nous faut une révolution pédagogique puisque justement, c'est l'enseignement qui fait que nous ayons l'esprit très tôt domestiqué.

Du reste, ici même nous avons créé un petit groupe d'enseignants et chercheurs sur 
le thème de "Reliance en complexité ", dans lequel nous avons une enseignante d'une école privée qui enseigne les petits dès le primaire et qui montre qu'effectivement, l'enseignant a beaucoup plus à apprendre de la complexité des enfants que de leur apprendre. Pourquoi ? Parce que l'esprit humain est fait pour pouvoir essayer de relier les choses les unes aux autres, ce que dans le fond la domestication casse un peu, et casse beaucoup plus encore la curiosité universelle. Donc : révolution pédagogique. Et si je la place sous l'étendard du mot complexité, je veux dire par là, que ce n'est pas seulement qu'il nous faut essayer d'apprendre à connaitre la pensée complexe, mais aussi qu'il nous faut aussi proposer des thèmes d'enseignements absents, aussi bien du secondaire que du supérieur, et qui sont nécessaires, à mon avis, pour affronter les problèmes de vie de la personne et du citoyen. Je l'ai fait dans ce petit livre qui s'appelle les Sept savoirs ${ }^{11}$ que vous connaissez, sans doute. D'ailleurs j'aurais souhaité qu'un ministre commence à introduire dans les programmes le premier savoir, la connaissance de la connaissance. Actuellement on commence à parler de métacognition, ce qui n'est déjà pas si mal, mais je trouve que le terme "connaissance de la connaissance " implique une réflexivité du mot connaissance sur lui-même, implique une sorte de boucle récursive, que le mot de métacognition ne possède pas, et qui serait assez utile puisque la métacognition est elle-même une cognition. Donc si vous voulez il y a une sorte de vice, parce qu'une métacognition qui ne soit pas une cognition, qu'est-ce que c'est? Autant j'apprécie ce que les neurosciences, du moins la partie qui s'intéresse au fonctionnement cérébral, peuvent apporter, autant je pense que c'est un apport nécessaire et insuffisant, et qu'il faut faire appel à autre chose. La connaissance de la connaissance, ce n'est pas seulement ce que peuvent nous apporter les connaissances sur le fonctionnement cérébral, c'est aussi la convergence des différentes connaissances acquises dans de multiples champs. Le problème de la perception par exemple, qui peut se trouver trompeuse, le problème de la théorie, elle-même, qui peut se trouver mutilante et tronquée, surtout quand cette théorie elle-même réduit la connaissance au simple calcul.

Nous avons donc des sortes multiples d'erreurs et d'illusions que nous pouvons débusquer dans la connaissance de la connaissance, tout en incitant à une connaissance plus pertinente capable de permettre de relier en donnant les éléments, les principes, capables de dépasser la pensée des seuls éléments. Ce que j'ai appelé « Méthode ", ce n'est pas une méthodologie, une programmation qu'il faut appliquer. C'est une façon de permettre à l'esprit de modifier sa vision des choses et de pouvoir prendre l'initiative de connaitre et de penser. C'est pour ça que même ce mot de méthode qui, si on prend ses origines, signifie un chemin, est transformé en programme parce que la façon dominante de penser, c'est de vouloir tout programmer, et non pas de jouer sur la stratégie qui nécessite des initiatives personnelles. Aujourd'hui d'ailleurs dans le fond, le mythe de l'algorithme maitre, le mythe de l'intelligence artificielle, qui est par ailleurs une chose très utile, c'est de penser qu'une machine pensante artificielle peut harmoniser et régler tous les rapports humains et sociaux. Donc on est de plus en plus dans l'illusion et dans l'erreur.

Révolution pédagogique : introduire la connaissance de la connaissance, introduire l'humain. C'est absolument paradoxal qu'on n'enseigne pas ce qui fait notre propre nature, notre propre qualité d'être humain. Et pourquoi ne le fait-on pas? Exactement pour les raisons que j'ai dites. Pour comprendre l'humain, il faut 
comprendre que l'humain est une trinité, c'est à dire un trio indissoluble entre l'espèce biologique humaine, la société humaine et l'individu humain. J'ai développé tout cela assez abondamment, pas seulement dans Le paradigme perdu mais dans mon livre l'Humanité de l'humanitét ${ }^{12}$. Et c'est à ce moment-là, qu'on peut coordonner et organiser tous les éléments de connaissance dispersés. Je reprends la phrase de Heidegger, jamais on n'a eu autant de connaissances sur l'homme et jamais on n'a su moins ce que c'est parce que toutes ces connaissances sont dispersées. Et en effet on a acquis des connaissances formidables sur la préhistoire, l'hominisation, les sociétés archaïques... Vous voyez, enseigner la connaissance de la connaissance, enseigner l'humain, enseigner la compréhension d'autrui, chose capitale... Je ne fais pas là le catalogue de mes sept savoirs. Mais, je le répète, tout ceci permet d'avancer vers la révolution pédagogique nécessaire. Plus importante, ou au moins aussi importante que fut la révolution pédagogique de Humboldt au début du $19^{\mathrm{e}}$ siècle, qui introduit dans l'université et donc dans l'enseignement, les sciences, qui à l'époque étaient naissantes, il n'y avait que la médecine. Vous voyez c'est ça qui, à mon avis, est l'essentiel.

GUY BERgeR : Au cours de ce parcours, vous avez rencontré, et je crois que vous rencontrez encore, un certain nombre de travaux de pédagogues, de mouvements pédagogiques, qu'on désigne souvent sous le nom d'éducation nouvelle, et qui correspondent à un certain nombre de mouvements du type de mouvements créés par Madame Montessori, Decroly, Freinet etc. En même temps, je voudrais interroger plus profondément ce rapport, parce qu'il introduit un terme nouveau, dans ce que vous nous avez dit, non pas au sens prétentieux d'une découverte mais il déplace un peu les notions. Vous avez énoncé au début que vous proposiez une révolution intellectuelle dans la manière de penser, dans la manière de connaitre. Avec le problème de ces mouvements pédagogiques, s'adressant en particulier à de jeunes enfants, c'est un autre mot qui devient dominant, c'est la manière d'apprendre. Et je crois qu'à partir du moment, où on introduit cette notion d'apprendre, d'autres aspects, je dirais de votre œuvre, prennent sens et deviennent éclairants. La première, c'est qu'on n'apprend pas sans désir, on n'apprend pas sans envie, on n'apprend pas sans plaisir. Et de ce fait, le lien, entre ce qui sera plus tard connaissance et qui est dans l'apprendre avec l'émotion, l'affectivité, le désir, sous toutes les formes de désir, est fondamental. L'autre chose qu'introduit je crois cet apprendre c'est que le premier geste d'apprendre, et le mot prendre n'y est pas par hasard contenu, c'est le geste moteur. Apprendre c'est d'abord toucher, c'est d'abord manipuler, c'est d'abord renverser, émietter, écraser, pour voir quelle est la consistance de cet objet, et l'acte d'apprendre introduit inévitablement le corps. Je crois donc que dans ce qui sera la matrice et de la pensée et de la connaissance et qui est l'apprendre, le corps est essentiellement présent. Je crois aussi que, à travers l'importance que vous aviez donnée au modèle biologique, mais aussi tout au long de vos travaux, ce corps est profondément présent. Et puis, toujours dans l'apprendre, parce que, par définition, on apprend ce que l'on ne sait pas, l'apprendre, c'est le rapport à l'inconnu, c'est le rapport à l'imprévisible etc. Ces trois thématiques me semblent certes présentes dans votre œuvre mais, je crois, se rencontrent de façon particulièrement pertinente dans la relation de cette œuvre avec ce qui se passe dans une école.

Edgar Morin : Sur ce que vous venez de me dire, je n'ai pu que formuler deux idées, deux mots. Premièrement, j'ai toujours répété que, pour enseigner, il faut de l'éros, j'ai repris la formule de Platon. Un enseignant qui n'a pas l'amour, la passion, pas seulement de ce qu'il enseigne, mais des enseignés, un enseignant qui ne fait que débiter, qui n'est qu'un fonctionnaire du savoir, ce n'est pas un enseignant. Il y a une mission enseignante, qui nécessite de se dédier à la chose, comme se dédient sur le plan religieux les missionnaires, c'est à dire quelque chose qui vous possède en entier. Et en effet quelle est cette mission? C'est peut-être la plus importante de 
toutes, de faire en sorte, en prenant de jeunes enfants, qui ont toutes les possibilités humaines les pires comme les meilleures, toutes les potentialités de l'homo demens comme de l'homo sapiens, que sorte le meilleur d'eux-mêmes à la fois affectivement et intellectuellement. Et la deuxième chose, que j'ai aussi comprise grâce à l'œuvre de Damasio $^{13}$, c'est qu'il n'y a pas de raison froide et glacée mais toujours liée à une émotion. J'ai toujours pensé que l'important est de jouer la dialectique entre la raison et l'émotion. Avec l'émotion il y a toujours un petit contrôle de la raison, mais la raison est mue aussi par le fait émotionnel, l'amour la sympathie etc. Et effectivement vous avez raison, il y a beaucoup d'initiatives pédagogiques, en France comme ailleurs, qui considèrent ces choses très importantes. J'ai été invité en juin dernier par un réseau pédagogique brésilien, qui lui-même avait mis au centre de son enseignement, l'enseignement à travers l'émotion. Ce qui les intéressait c'était de complémentariser cet enseignement émotionnel par un enseignement à la complexité. Devant 1500 enseignants brésiliens, j'ai pu dire comment les choses peuvent se combiner. Je participe tout à fait à ça. Je n'enseigne pas à des enfants, ni à l'université, mais je donne mon apport. D'ailleurs prenez Montessori, il s'ouvre à Rennes une école Montessori-Morin ${ }^{14}$, preuve que tout ceci peut très bien s'associer. La chose importante aussi, c'est que justement il faut laisser la liberté à la curiosité polymorphe de l'enfant. La tragédie de l'adultération, c'est que cette curiosité va se rétrécir, se compartimenter sur un secteur de la vie et du monde, et ignorer le reste. C'est évidemment cette curiosité infantile qu'il faut sauvegarder adolescent, adulte, et même si possible vieux. Donc là aussi, je pense que je vais exactement dans le même sens, mais en insistant sur le fait que déjà l'enfant possède en lui ces aptitudes et que malheureusement l'éducation joue un rôle castrateur. En fait je dirais un autre mot, je dirais que l'éducation doit favoriser l'auto-éducation, c'est-à-dire que l'éducation doit être un phénomène auto-hétéro-productif. L'enseignant aide l'enseigné à développer ses facultés naturelles. De mon expérience, j'ai eu quelques professeurs qui m'ont passionné parce qu'ils étaient passionnés. Et quelques professeurs qui m'ont ennuyé parce qu'ils étaient ennuyés. Bon. Mais je me suis fait ma culture en combinant ma propre recherche à celle que m'apportait l'école. Par exemple c'est tout seul, que j'ai découvert les auteurs qui me convenaient. A l'époque pour moi à treize ans, c'était Anatole France, puis après c'était Montaigne, et puis c'était Dostoïevski, c'était Tolstoï. Mes propres vérités je les ai accueillies tout seul, Rimbaud je l'ai trouvé tout seul! Je veux dire aussi qu'il faut aider la recherche par chacun de ses propres vérités, que chacun découvre la vérité qu'il attendait, et qu'il ignorait attendre, c'est cela qui me semble tout à fait essentiel. Donc je conclue, complémentarité absolue entre ce que vous dites et ce que moi je vous propose.

GUY Berger : Oui. Tout à fait. Et en même temps, dans ce que vous venez de dire et en particulier à la fin, vous apportez quelque chose à un débat alors, qui est typiquement français, vous le savez, qui est ce débat actuellement très médiatisé et très stupide, entre ceux qu'on présente comme des pédagogues, et pédagogues et démocrates, et qu'on oppose à des républicains, détenteurs, gardiens fidèles de la culture et soucieux de la transmission. Je crois que ce débat entraine des contresens et vous y répondez. Le premier contresens il est lié à la langue française, et il est frappant que quand on parle d'apprendre on pense aussitôt enseigner. En français, le verbe enseigner se dit aussi bien de l'enseignant qui enseigne les élèves, que de l'enseignant qui enseigne les mathématiques, c'est le fameux double accusatif de nos enfances pour ceux qui ont fait du latin. D'autre part dans le mot apprendre, apprendre c'est ce que fait l'élève, et pourtant j'apprends aux élèves quelque chose. Ce qui me frappe dans ce que vous dites en fait, c'est que vous êtes parti de l'idée de l'enseignement de la complexité, de l'enseignement de vos enseignants 
eux-mêmes passionnés etc. En fait une des choses qui oppose quelquefois les pédagogues modernes dits justement de l'école nouvelle, aux pédagogues traditionnels, c'est que les uns considèrent que seul le mouvement de l'enfant vers la connaissance est porteur de connaissance, et que tout acte de transmission serait en quelque sorte une manière de l'enfermer dans des savoirs préfabriqués, disciplinarisés et disciplinés par ailleurs, et que d'autre part au fond, c'est cela que récusent les républicains en disant on ne fait que transmettre. Ce qui me frappe dans ce que vous avez dit, et en ce sens vous ne coïncidez pas avec un certains discours un peu dogmatique de l'école nouvelle, l'apprendre est fondamental, c'est qu'il n'y a pas de connaissance sans qu'il y ait aussi transmission. C'est un équilibre, de cet apprendre et de cette transmission qui est la chose importante. Ce qui pose un problème, un conflit de vérité, ce que j'apprends par moi-même et ce que je transmets n'est pas nécessairement homogène. Si bien, que j'ai envie de déplacer un peu notre discussion. Nous sommes partis de la notion de complexité. Je crois que, aussi bien quand vous avez parlé du rapport émotion rationalité, à partir de Damasio, que dans ce conflit possible entre l'apprendre et le recevoir, une autre notion importante qui vous est chère et qui ne se confond pas avec celle de complexité est celle de dialogique. C'est-à-dire, celle d'un maintien permanent d'une tension entre des forces contradictoires, qu'il ne s'agit pas de résoudre. II ne s'agit pas de trouver une solution, même pas une synthèse, il s'agit de faire de cette tension un moteur, un déclencheur au fond, d'une démarche qui ne finit jamais. Et je serais heureux que vous me parliez un peu de ce rapport au dialogique d'autant plus qu'il y a eu un tel succès du mot dialectique qu'il a écrasé en quelque sorte cette notion de tension, peut-être au risque de la faire disparaitre.

Edgar Morin : Effectivement on peut dire que la dialogique est une façon d'hériter d'une tradition, très minoritaire dans notre monde occidental, mais dont le premier représentant et le plus significatif à mon avis demeure Héraclite. A à la différence de ceux qui plus tard vont être des dialecticiens comme Hegel pour lequel les contradictions peuvent être toujours surmontées par une synthèse, mais pour qui quand même cette synthèse elle-même est un terme contradictoire. Si on est fidèle à Hegel et l'hégélianisme il n'y a pas de terminus, il y a le mouvement perpétuel mais il y a toujours le dépassement. Alors qu'avec Héraclite nous vivons pleinement une contradiction. Héraclite dit : «éveillés nous dormons ». On n'en sort pas. On est à la fois éveillé et dormeur. Héraclite dit «concorde et discorde sont pères et mères de toutes choses", ce qui veut dire qu'on ne peut plus dissocier la concorde de la discorde, on est au cœur de ce que j'appelle la dialogique, la nécessité d'associer des notions qui sont en elles-mêmes antagonistes et pourtant inséparables. Dès qu'on entre un peu profondément dans des domaines de réalités, humaines ou du monde extérieur, nous trouvons des contradictions insurmontables. Que ce soit sur l'origine de l'univers qui naitrait d'un vide qui ne serait pas totalement vide, d'un temps qui naitrait du non-temps, de l'espace etc. On a des contradictions sur la nature du réel, que l'on a d'ailleurs vu apparaitre grâce à la physique quantique, où toutes nos notions classiques de temps, d'espace, d'objet, se sont trouvées démantibulées. Il y a des domaines où effectivement nous devons dépasser, transgresser la logique classique, c'est cela que j'appelle la dialogique. Parfois nous pouvons trouver des synthèses, parfois nous n'en trouvons pas et nous faisons des paris! Je reprends ce que disait Bateson, qui sans employer le mot dialogique, parlait de double bind ${ }^{15}$ : quand deux impératifs contraires arrivent en même temps à un esprit, que doit-il faire? Il fait un pari. Il décide soit de ne rien faire, soit de faire ceci ou cela. Nous sommes condamnés, là aussi c'est très important à enseigner, au pari, toute décision est un pari. C'est aussi ça la complexité, parce que dans l'éthique simple, celle que disait Kant où effectivement il suffit d'avoir de très bonnes intentions, finalement la conséquence de bonnes intentions est souvent infernale. Que j'ai connues de bonnes 
intentions où les gens qui croyaient travailler pour le bien de l'humanité faisaient le contraire, on voit cela sans arrêt. Le problème là aussi est de comprendre qu'une fois qu'on prend une décision, on fait un pari, l'action que l'on décide d'entreprendre se trouve dans un jeu d'interactions ou de rétroactions qui va la dévier et parfois lui revenir sur la tête. Donc si vous voulez tout ça fait partie de la révolution intellectuelle, tout ça fait partie des choses capitales qui nous permettront d'affronter les grands défis de notre temps. La dialogique pour moi est un principe essentiel. Bien entendu, quand vous découpez la réalité en petits morceaux, chaque petit morceau obéit impeccablement à la logique classique de la noncontradiction. Mais dès que vous cessez de couper la réalité en petits morceaux, vous voyez apparaitre les insuffisances et les limites de la logique, comme celle de la rationalité. Ce sont des éléments très très importants. Je dirais que la dialogique, le principe de la boucle, qu'elle soit rétroactive et récursive, c'est la rupture avec la pensée linéaire. Et enfin il y a ce que j'ai appelé le principe absolument illogique de l'hologrammatique, c'est-à-dire que non seulement une partie se trouve dans le tout mais que le tout est à l'intérieur de la partie. Tout ceci va permettre de comprendre la complexité, et de mieux affronter la complexité.

GUY BERgER : En présentant et en nous aidant à comprendre votre conception du dialogique, vous avez utilisé le mot de pari, et évoqué Bateson. Il est évident que pour nous français, le pari c'est Pascal. Et il me renvoie aussi à la vision pascalienne qui est la vôtre et qui fait que vous prendriez volontiers à votre compte sa célèbre affirmation «s'il se vante je l'abaisse s'il s'abaisse je le vante et je le poursuis sans cesse jusqu'à ce qu'il reconnaisse qu'il est un monstre incompréhensible... », mais la dialogique dans ce que vous en dites n'est pas simplement quelque chose qui s'applique au mouvement de la connaissance, j'ai l'impression qu'elle est en fait fondamentale dans votre conception même de l'homme, sapiens/demens, que l'homme est dialogique dans ce qu'il est, qu'il est contradiction, qu'il est tension, qu'il est tension et qu'il est bataille en quelque sorte avec lui-même...

Edgar Morin : Bien sûr, oui. C'est faber l'homme de la pratique concrète et c'est l'homme mythologique et religieux en même temps, c'est le même à la fois homo économicus, et homo ludens qui joue, qui est la gratuité.

GUY BERger: Autrement dit le dialogique n'est pas seulement une affaire de la connaissance, le dialogique est l'anthropologique lui-même, me semble-til.

Edgar Morin : Exactement !

GUY BERgER : Et ceci est quelque chose qui correspond au lecteur de votre œuvre que je suis, que beaucoup d'entre nous sont, c'est que si effectivement vous avez commencé par nous réveiller de nos sommeils dogmatiques, pour évoquer des phrases célèbres nous enfermant effectivement dans nos apprentissages adultes...

Edgar Morin : Ou plutôt je n'ai pas réussi à vous réveiller !

GUY BERgER : En tout cas personnellement...

Edgar Morin : Pour vous d'accord...

AUgustin MUTUALE : Pour nous aussi !

GUY BERgER : Vous êtes devenu pas simplement quelqu'un qui nous aide, nous bouscule, nous secoue, et nous emmerde quelques fois, dans le champ de la connaissance, mais aussi quelqu'un qui nous propose une conception de l'homme. Et je dirais que c'est tout à fait important que dans la façon même dont on vous présente on ait cessé de parler de vous comme sociologue ce qui correspond à ce que furent vos premières œuvres, votre travail sur Plozévet ${ }^{16}$, La rumeur d'Orléans ${ }^{17}$, Les stars $^{18}$, pour vous présenter comme philosophe. C'est une manière peut être de donner trop de valeur à la philosophie mais cela c'est une autre question, et il ne s'agit pas de vous. Mais c'est une conception de l'homme 
et en tant que conception de l'homme, ça fait apparaitre quelque chose de nouveau et qui est important aussi vis-à-vis de l'éducation, qui n'est pas simplement une réflexion sur le connaitre, mais une réflexion sur l'éthique. Et je pense que dans le mouvement, qui est allé du premier systémisme, "rez de chaussée de la complexité », comme vous le disiez avec Ardoino dans une série d'entretiens, vers le dialogique, vers justement ce travail sur la tension anthropologique d'un homme émotif et rationnel, on entre dans une démarche qui est UNE ÉThique et finalement qui devient UNE Politique. Et c'est peut-être ce passage, qui est aussi le problème de l'éducation, où tout apprentissage est en même temps éthique et politique et que l'on peut lire dans vos dernières œuvres et vos dernières interventions.

Edgar Morin : Je dirais que la phrase clé à laquelle j'ai essayé de travailler toute ma vie, c'est la phrase de Kant: "Que puis-je savoir, que puis-je croire, que puis-je espérer ». Et pour répondre à ces questions il faut que je sache ce qu'est l'homme. Donc pour moi la question de l'anthropologie, disons puisque ce mot est trop limité aujourd'hui, de l'humanologsime, la question de l'humain est devenue une question centrale pour savoir ce qu'il faut connaitre. C'est une question en boucle, parce que plus je connais des choses sur l'humain, plus je peux enrichir ma façon de connaitre, et ma façon de connaitre plus je l'enrichis.... Si vous voulez, il y a un rapport inséparable et en boucle entre ces trois éléments. Je dirais, bien que l'éthique soit quelque chose de normatif, que pour moi l'éthique, puisque j'ai fait un dernier volume dans la méthode là-dessus, est une chose dont je n'ai pas essayé de formuler les règles, mais dont j'ai voulu connaitre les sources. Et les sources, elles sont à la fois anthropologiques, biologiques et sociales. Solidarité/responsabilité. Vous trouvez ça déjà dans les sociétés de mammifères, vous les trouvez dans toutes les sociétés humaines. C'est biologique dans des espèces qui existent avant la nôtre. C'est un phénomène sociologique, la société contribue à cela. C'est un principe égocentrique, que nous avons en nous, qui nous pousse à défendre notre propre intérêt vital. Et c'est un principe d'ouverture sur un nous qui nous inscrit dans une communauté, et dans cette inscription nous avons la solidarité et la responsabilité. Si vous voulez, l'éthique se dégage et par là même nous comprenons même ses problèmes. Ce que j'ai voulu comprendre, c'est la problématique éthique, ce sont les contradictions éthiques, les devoirs contradictoires comme le médecin qui a affaire à un agonisant et doit savoir s'il faut le débrancher ou non. Vous avez les incertitudes éthiques, vous avez même les aveuglements éthiques, vous avez toutes ces choses-là. J'ai voulu explorer ce champ-là, que bien entendu l'on peut considérer dans l'enseignement. Si vous introduisez le processus de la mondialisation qui est l'étape actuelle de l'ère planétaire, à ce moment-là vous introduisez nécessairement la communauté de destin humaine face à tous les périls auxquels nous sommes de plus en plus confrontés maintenant de par notre développement techno-socio-économique. De là vont se dégager quelques principes d'action politique, que moi-même j'ai pu définir dans un livre qui s'appelle $L a V V_{i e}{ }^{19}$ et que j'avais commencé à définir dans un livre plus limité qui s'appelait Pour une politique de civilisation ${ }^{20}$, qui consistent à lutter contre les dégradations de notre civilisation notamment en ce qui concerne les solidarités et les fraternités, processus dû aux qualités et aux défaut de notre individualisme, à la compartimentation, à la bureaucratisation etc. Je ne dis pas qu'on peut déduire logiquement une éthique d'une conception de l'homme et une politique de l'éthique, mais elles communiquent. Et elles nous montrent aussi toujours la voie dans laquelle elle est. Sans faire une œuvre réductrice, qui réduise, la complexité est pour moi une chose qui permet d'ouvrir et jamais de refermer. 
GUY Berger : J'avais envie de continuer, en disant ce que j'apprends de "mon Morin ». Je suis frappé, justement quand vous parlez de complexité, qu'en même temps vous y introduisez je dirai une profonde dialogique interne.

\section{Edgar Morin : C'est ça, j'identifie la dialogique à la complexité !}

GUY BERgER : Qu'on découvre quand on lit par exemple les Sept principes qui concluent en quelque sorte votre livre Tête bien faite, on a bien sûr une complexité combattive, mais qui par certains côtés est apollinienne, sereine. II y a là quelque chose justement de l'ordre de la prise en compte des parties et du tout, de la prise en compte d'une perspective globale, d'une perspective qui essaie de tenir compte de tous les aspects de la réalité. D'ailleurs c'est tout à fait intéressant que le septième principe que vous évoquez introduise le sujet connaissant dans la connaissance. C'est intéressant parce que du point de vue du pédagogue justement, c'est le premier. Dans l'apprendre, c'est le sujet qui connait qui est fondateur. Et on pourrait presque, du point de vue pédagogique, faire une lecture inversée de ces sept principes, le septième devenant en quelque sorte le premier. D'autre part, partant d'une démarche qui est éthique, on arrive je dirais à un monde violent, angoissant, inquiétant, dont je trouve l'image qui dans mon histoire m'a le plus marquée dans cette œuvre que vous avez composée avec quelqu'un d'autre, qui est Terre-Patrie ${ }^{27}$. J'ai été extrêmement frappé, avec d'autant plus de plaisir et une malice que je réserve à mes collègues qui sont tous membres de l'Institut catholique, par votre chapitre dernier s'appelle l'Evangile de la perdition. Et il commence d'ailleurs comme sous-chapitre par le titre « La perte du salut, l'aventure inconnue ». C'est-à-dire vous êtes à la fois, porteur de réponse, mais d'une réponse qui est porteuse de questions, et d'une question qui se veut en fait intensément sans réponse, car vous continuez, je dirais presqu'avec une véritable cruauté, par parler de la bonne-mauvaise nouvelle, et quand on sait que l'évangile c'est justement la bonne nouvelle, dire que la bonne mauvaise nouvelle est brusquement captée dans vos filets, une pensée qui n'était pas forcément la vôtre. Et dans votre conclusion vous proposez en quelque sorte six principes de vie qui à mes yeux sont extraordinaires, y compris dans cette mise au regard des sept principes de la connaissance.

Le premier est un principe vital, « de même que tout ce qui vit s'auto-régénère dans une tension incoercible vers son futur, de même tout ce qui est humain régénère l'espérance en régénérant son vivre, ce n'est pas l'espérance qui fait vivre, c'est le vivre qui fait l'espérance ». C'est-à-dire en affirmant un vitalisme pour employer un terme un peu grossier, un peu simpliste mais tout à fait fondamental. Le second est le principe de l'inconcevable, toutes les grandes transformations et créations ont été impensables avant qu'elles soient produites. Le troisième est le principe de l'improbable. Le quatrième est le principe de la taupe qui creuse ses galeries souterraines et transforme le sous-sol avant que la surface en soit affectée. Le cinquième c'est la prise de conscience du danger. Et le sixième vous le dites est anthropologique, c'est à dire il pose en quelque sorte cette espèce d'explosion de l'homme dans son rapport avec une ère planétaire.

C'est-à-dire, comment porter à la fois, un principe de réponse, que j'ai prétendue, je le rappelle, apollinienne, et qui par certains côtés nous aide à survivre, et une angoisse de vivre, et une affirmation de l'inconnu, et une espèce d'appui en quelque sorte sur l'improbable, et donc sur l'incertitude qui est en quelque sorte comme un appel à la vigilance. Or c'est ce double message, qui est formateur, qui est angoissant aussi, et qui me semble là encore, compris dans la notion de complexité, mais introduisant dans la conception de la complexité quelque chose d'absolument original par rapport à tout ce qu'on en dit.

Edgar Morin: Tout d'abord, dans ce livre, et c'est ce qui a déplu à pas mal de lecteurs, cet évangile de la perdition, je commence par dire nous sommes perdus. Nous sommes perdus, d'abord dans cet univers, sur cette petite planète d'un soleil de banlieue d'une galaxie périphérique. On est perdu. Nous ne savons pas pourquoi nous sommes nés, nous ne savons pas pourquoi nous allons mourir. Alors dans ce principe de perdition se trouve en quelque sorte le réconfort. C'est-à-dire, ne disons pas soyons frères pour que nous soyons sauvés, soyons frères parce que nous sommes 
perdus. C'est ce qu'on trouve un peu dans le livre d'Albert Cohen Belle du seigneur, c'est un peu cette idée, si nous sommes conscients que nous sommes tous mortels, tous sujets au malheur à la tristesse, nous fraterniserons, nous le devrions, fraterniser. Si nous prenons conscience non pas seulement de la grandeur humaine qui lance des fusées des missiles dans le ciel, mais où le plus puissant des despotes comme Staline disant le vainqueur ce sera la mort, vivant dans l'angoisse... Si nous sommes conscients de tout cela, fraternisons! C'est un peu cette idée que je maintiens encore bien qu'elle déplaise.

Parce que pour moi l'espérance est inséparable d'une certaine désespérance, elle est dans la vie... Alors ça me fait penser, je ne sais pas si je le cite, à cette pièce du grand romantique hongrois, Madach, la Tragédie de l'homme. C'est une pièce en plusieurs tableaux, ça commence dans le jardin d'Eden tout est merveilleux entre Adam et Eve, et puis elle mange la pomme, catastrophe. Ensuite ça continue, c'est Cléopâtre et Marc-Antoine, ils sont heureux ils s'aiment, Jules César, catastrophe. Tout commence bien et tout se termine mal. L'avant dernier tableau se passe sut la terre refroidie en l'an 4000 etc, le soleil a pali, il ne reste plus qu'un couple d'esquimaux sur leur igloo. Ils sont très très tristes évidemment, et puis la femme accouche! Et quand l'enfant sort ils sont heureux, ils sourient, ils ont l'espérance. Le vivre donne l'espérance. Et ce qu'il y a de très bien dans cette pièce c'est que il y a un épilogue où Dieu parle, à un moment donné il s'adresse à Satan, « reste Satan, j'ai besoin de toi, j'ai besoin de ta négativité, sans toi... » Et finalement on arrive à la dialogique nécessaire entre les forces de négation et les forces au contraire d'affirmation et d'amour. Cela je le donne métaphoriquement, c'est ma façon d'être aussi, de penser. Parce que le message principal de ce livre Terre patrie c'est celui que je vous ai dit. Désormais, nous avons une patrie terrestre qui recouvre nos différentes patries sans lignée, et dans le fond, si nous en prenons conscience, nous sommes à même de mieux résister au péril qui nous menace. Cela me semble du bon sens. Voilà.

Le dernier mot c'est que, effectivement, par rapport à toute conception statique, on dit «ah, oui, une société meilleure... » Je crois qu'il n'y pas de terminus historique. Peut-être la mort de l'humanité, on ne sait pas. Nous sommes dans une aventure inconnue. Le cours de l'aventure a toujours été inconnu, à chaque étape. A l'étape hominienne on ne savait pas que la bipédie amènerait au langage et à la culture. $\mathrm{A}$ l'époque préhistorique on ne savait pas qu'il y aurait des machines et l'intelligence artificielle. A la fin de l'empire romain, impossible de concevoir la Renaissance. Aussi bien impossible de concevoir que le message de Bouddha, de Mahomet, de Jésus, tout ça après une incubation, seraient des grands événements.... Ce sont toujours des petites déviances qui produisent de grands mouvements historiques, c'est l'improbable. Je crois que c'est ça les leçons de l'histoire. Et je crois aussi que l'histoire, sa grande leçon est qu'elle doit faire copuler Karl Marx et Shakespeare. C'est à la fois une histoire qui a des forces de rationalité, et de délire. Une histoire racontée par un idiot, pleine de bruit et de fureur, et qui ne signifie rien. Il faut être conscient de tout ce mélange de rationalité et d'irréalisable, c'est cela la vie dans laquelle nous sommes lancés. Mon idée, ce n'est pas d'apporter des choses consolantes, mais des choses qui aident, à affronter, à affronter l'angoisse, à affronter la mort, à affronter les douleurs de la vie. C'est cela qui me semble important, et où la révolution intellectuelle et pédagogique est, aujourd'hui, un élément clé.

AUgustin mUtUale: Je vais rapidement prendre la parole. J'ai aussi lu beaucoup lu Dostoïevski, j'ai développé le concept de la responsable liberté créatrice, mais aussi j'ai 
travaillé sur le pari de Pascal et sur Kierkegaard que vous présentez en grand au niveau de la rencontre. Ce que je voudrais ramener de vous aussi pour la faculté que je dirige, c'est cet évangile. Quand j'ai lu Terre-patrie je me suis retrouvé en face de cet évangile. Au départ, bien sûr je suis chrétien, cela m'a un peu déboussolé et petit à petit je me suis mis, avec Dostoïevski, à sidérer. En disant ce que vous êtes en train de dire, là, quand Dostoïevski dit c'est là où il n'y a plus rien, là où il y a le désespoir, qu'on rencontre la plus grande espérance. Et donc j'aimerais vous entendre sur quelque chose que je mets en place actuellement, un projet facultaire qui traite de la communauté éducative inclusive. Cela fait écho à vos questionnements, puisque la question de l'écologie c'est vraiment votre question, et la question de l'autre, c'est aussi votre question. Vous parlez de toutes ces différentes rencontres de ce monde, de notre monde, et j'aimerais vous entendre sur la question du faible. Puisque dans cette régénération nous ne sommes pas dans ce monde de Nietzsche où il y la régénération par la puissance, mais notre régénération nous apporte aussi des plus faibles, qui sont là avec nous. En éducation, quelle est la place du faible? Quelle est la place que vous donnez dans ce monde qui nous est en commun, la place du faible dans l'éducation? Comment être avec celui qui est plus faible que nous, et que nous, nous nommons dans la pédagogie inclusive, mais quels sont vos mots, quelle est la place? Quel est ce monde que cet évangile en perdition peut donner à celui qui nous est faible? Comment sauver le monde avec les faibles?

Edgar Morin : Vous avez déjà cet implicite dans le terme du message évangélique, laïcisé comme je le fais, est un message qui s'adresse dans le fond aux humiliés, aux faibles, aux plus petits, à la sagesse de ceux qui ignorent tout... Moi je crois que du point de vue pédagogique, l'enseignant n'a pas tellement à s'occuper des élèves qui sont brillants, qui réussissent tout seuls, sa mission est beaucoup plus d'aider ceux qui ont des difficultés, ceux qui ont des perturbations personnelles, des faiblesses. Autrement dit je pense que tout ce qui est fort doit aider le faible. Cela me semble être un principe de base. Ce l'est pour moi.

FABIENNE SERINA-KARSKY : Merci cher Edgar de nous avoir reçus, merci pour tout ce que vous nous avez apporté dans cet entretien dont Guy Berger s'est fait le porte-parole avec brio. J'espère que nous pourrons continuer une prochaine fois.

Edgar Morin : Je voulais vous remercier car vous m'avez entrainé sur des sujets importants, enfin qui pour moi sont très importants. J'ai été content de dialoguer avec vous, et la prochaine fois nous irons un peu plus loin.

\section{BIBLIOGRAPHIE}

Barel, Y. (1979). Le paradoxe et le système. Grenoble : Presses Universitaires de Grenoble.

Berger, G., Courtois, M., Perrigault, C. (2015). Folies et raisons d'une Université Paris 8, de Vincennes à Saint-Denis. Paris : Editions Petra.

Damasio, A. (1995). L'erreur de Descartes : La raison des émotions. Paris : Odile Jacob.

Lesourne, J. (éd.) (1979). La notion de système dans les sciences contemporaines. Aix en Provence : Ed. de la librairie de l'Université.

Morin, E. (1967). Commune en France. La métamorphose de Plodémet. Paris : Fayard. 
Morin, E. (1973). Le paradigme perdu. La nature humaine. Paris : Seuil.

Morin, E. (1969). La rumeur d'Orléans. Paris : Seuil.

Morin, E. (1972). Les stars. Paris : Seuil.

Morin, E. (1999). La tête bien faite. Repenser la réforme, réformer la pensée. Paris : Seuil.

Morin, E. (1999). Les sept savoirs nécessaires à l'éducation du futur. Paris : Seuil.

Morin, E. (2001). La Méthode, tome 5 : l'Humanité de l'humanité. L'identité humaine. Paris : Seuil.

Morin, E. (2002). Pour une politique de civilisation. Paris : Arlea.

Morin, E. (2011). La Voie : pour l'avenir de l'humanité. Paris : Fayard.

Morin, E. \& Kern, A.-B. (1993). Terre-patrie. Paris : Seuil.

Mukungu Kakangu, M. (2009). Vocabulaire de la complexité- Post-scriptum à La Méthode d'Edgar Morin. Paris : L'Harmattan.

Peyron-Bonjan, C. \& Ardoino, J. (2000). Réforme de la pensée, pensée de la réforme - Entretiens avec Edgar Morin sur l'éducation. Pratiques de formation-Analyses, $\mathrm{n}^{\circ} 39$.

Rosnay, J. de (1975). Le Macroscope. Une vision globale. Paris : Seuil.

\section{NOTES}

1. Nous tenons à remercier à Danielle Banckaert pour son aide lors de la préparation à l'entretien ainsi que pour son accueil.

2. Peyron-Bonjan, C. \& Ardoino, J. (2000). Réforme de la pensée, pensée de la réforme - Entretiens avec Edgar Morin sur l'éducation. Pratiques de formation-Analyses, ${ }^{\circ} 39$.

3. Marius Mukungu Kakangu, M. (2009). Vocabulaire de la complexité- Post-scriptum à La Méthode d'Edgar Morin. Paris : L'Harmattan.

4. Rosnay, J. de (1975). Le Macroscope. Une vision globale. Paris : Seuil.

5. Barel, Y. (1979). Le paradoxe et le système. Grenoble : Presses Universitaires de Grenoble.

6. Lesourne, J. (éd.) (1979). La notion de système dans les sciences contemporaines. Aix en Provence : Ed. de la librairie de l'Université.

7. Morin, E. (1967). Commune en France. La métamorphose de Plodémet. Paris : Fayard.

8. Morin, E. (1973). Le paradigme perdu. La nature humaine. Paris : Seuil.

9. Berger, G., Courtois, M., Perrigault, C. (2015). Folies et raisons d'une Université Paris 8, de Vincennes à Saint-Denis. Paris : Editions Petra.

10. Morin, E. (1999). La tête bien faite. Repenser la réforme, réformer la pensée. Paris : Seuil.

11. Morin, E. (1999). Les sept savoirs nécessaires à l'éducation du futur. Paris : Seuil.

12. Morin, E. (2001). La Méthode, tome 5 : l'Humanité de l'humanité. L'identité humaine. Paris : Seuil.

13. Cf. i.e. Damasio, A. (1995). L'erreur de Descartes : La raison des émotions. Paris : Odile Jacob.

14. Le projet initial, dénommé "Campus Montessori-Morin ", a été changé en juin 2020 en la dénomination « l'Ecole du sens-Centre international de recherches pédagogiques ».

15. Double lien ou double contrainte.

16. Morin, E. (1967). Commune en France. La métamorphose de Plodémet. Paris : Fayard.

17. Morin, E. (1969). La rumeur d'Orléans. Paris : Seuil.

18. Morin, E. (1972). Les stars. Paris : Seuil.

19. Morin, E. (2011). La Voie : pour l'avenir de l'humanité. Paris : Fayard.

20. Morin, E. (2002). Pour une politique de civilisation. Paris : Arlea.

21. Morin, E. \& Kern, A.-B. (1993). Terre-patrie. Paris : Seuil. 


\section{RÉSUMÉS}

Cet entretien a pour objet de mettre au jour l'apport de la pensée complexe à l'éducation à travers la voix d'Edgar Morin, en dialogue principal avec Guy Berger.

The purpose of this interview is to bring to light the contribution of complex thinking to education through the voice of Edgar Morin, in a main dialogue with Guy Berger.

INDEX

Mots-clés : Edgar Morin, Guy Berger, éducation, pensée complexe, dialogique- humain

Keywords : Edgar Morin, Guy Berger, education, complex thought, dialogical- human

\section{AUTEURS}

\section{GUY BERGER}

Université Paris 8

\section{AUGUSTIN MUTUALE}

ISP-Faculté d'éducation, Institut Catholique de Paris, Unité de recherche « Religion, Culture et Société » : Langues, Cultures, Histoire et Education

\section{FABIENNE SERINA-KARSKY}

ISP-Faculté d'éducation, Institut Catholique de Paris, Unité de recherche « Religion, Culture et Société » : Langues, Cultures, Histoire et Education

\section{SÉVERINE PARAYRE}

ISP-Faculté d'éducation, Institut Catholique de Paris, Unité de recherche « Religion, Culture et Société » : Langues, Cultures, Histoire et Education 